\title{
OPTIMAL OPERATION OF PIPELINE TRANSPORTATION SYSTEMS
}

\author{
E. F. Camacho*, M. A. Ridao*, J. A. Ternero* \\ and J. M. Rodriguez**
}

*Dpto. Ingenieria, Electrónica, de Sistemas y Automática, Escuela Superior

Ingenieros Industriales, Universidad de Sevilla, Spain

**Dpto. Oleoductos-Operaciones, C.A.M.P.S.A., Spain

\begin{abstract}
This paper presents a simulator of an oil pipeline for scheduling purposes. The simulator includes an algorithm for optimizing the energy operating costs. The optimization algorithm works in two steps. The first one consists of the computation of a function that measures the estimated minimum cost to the goal node. This computation involves the use of Bellman's optimality principle and of some heuristic rules in order to avoid the combinatorial explosion. During the second step the optinum trajectory is obtained with the help of the function mentioned above and using an accurate simulation of the transportation system. The simulation considers those aspects which are relevant to the optimization problem and takes into account the following factors: topology and topography of the network, non-linear characteristics of pumps and pipelines, variable demands of consumers, time changing prices of electrical energy and hydraulic equations throughout the system. The simulator is being used by CAMPSA (the major oil distribution company in Spain) Some results obtained with an oil pipeline system in Northern Spain are presented in the paper.
\end{abstract}

Keywords. Computer Aplication; Simulation; Transportation control; Optimization; Dynamic progranming.

\section{INTRODUCTION}

One of the main ways of transporting oil products is by pipeline networks. The products are transported through the pipeline from the input nodes, normally refineries or ports, to the output nodes where they are stored in tanks. From there, they are transported to the final consumers by trucks.

As the products are injected into the system in different batches, at any given time clifferent products, separated by the corresponding interfaces. can be transported. The batches are routed through the network by opening or closing the appropiate set of valves.

The mechanical energy needed for transportation is supplied by a set of electrically powered pumps. The transportation velucity. for a particular network and a given pipeline state, depends on the number and the flow-head characteristics of the pumps working at each of the pumping stations.

The operation of a multi-fluid transportation system consists of determining the order and the way in which the clifferent fluids are going to be transported to satisfy the demand. This task can be divided into two different problems. The first one involves the determination of the approximate transportation needs. The solution of this problem will consist of

\footnotetext{
- \& batch sequence that will minimize the number of interfaces and will cover the consumption needs at each destination node.

- an approximate pumping schedule.
}

The batch sequence consists of a list of batches each of which is clefined by the product identification, approximate departure time, the volume destined to each output node and approximate arrival time to each terminal node.

The second problem involves determining how to set the different pumps and valves at each time interval so that the batch sequence given above is carried out in a given period of time. This can be accomplished in different ways, that is, using different pump combinations and valve positions ( and therefore flows at all points in the network) in the clifferent time intervals. This will turn into different batch arrival times, and different power operating costs as the price of electricity changes throughout the day and also friction losses and pump performances depend on the flow.

The power costs can be substantial in big pipelines and are increasing with respect to the total operating costs as shown in (Basavaraj, 1984). The interest of reducing or even better, optimizing. these costs is therefore evident.

The problem has been dealt with in the literature, especially for mono-fluid transportation systems, as is the case of water distribution systems (Coulbeck, 1978), (Millaoka 
et al. 1984). However, even in this case, the optimization problem leads to a dynamic and non-linear problem of high dimension as stated in (Kemplous et al. 1986). In this last reference an optimization algorithm that works at two levels has been proposed. At the upper level the dynamic problem is solved and some data passed to the lower level where the static problem is solved giving the number of pumps turned-on in the pumping station.

This article presents a simulator to be used by a pipeline scheduler in order to facilitate the operation of pipelines and optimize the power bill. The optimization algorithm has been developed by the authors and has been tested with models in a previous paper (Camacho et al, 1989). In this paper an application of the simulator to a real oil pipeline is presented.

\section{PIPELINE MODEL}

The main objetive of the model presented in this paper is to serve as a tool for schedulers. Given a pumping sequence and an initial state for the pipeline, the user should be able to simulate with reasonable accuracy the arrival time of the different batches at the terminals, the state of the pipeline at any given moment, and the evolution of the level at the terminals, in order to judge whether the pumping schedule fulfills the needs of the terminals. Only those aspects of the pipeline relevant to schedulers were considered. Bearing this in mind, a steady state model has been chosen.

This model is valid for using with pipeline networks with just one entrance point for the product (tree topology). Many real pipelines take this form. On the other hand, the normal method of operating more complex pipelines can be thougth of as various tree topology pipelines working together.

The topology of a pipeline network is defined by its component parts. The different parts included in the model are as follows:

- Pumping station: including the different component pumps and the way in which they are connected. Each of these pumps is associated to their corresponding characteristic curves Head-Flow and Power-Flow.

- Terminals : each of the terminals contains a set of storage tanks for the products and an out-let valve from the pipeline to the tanks. The capacity of each of these tanks must be known.

- Valves : maximum pressure valves, normally placed at the out-let of the pumping station.

\section{- Bifurcations}

\section{- Pipeline sections}

One aspect to be considered when making a model is defining the topographical profile of the ground, which is of great importance for calculating the pressure drop. The calculation of the hydraulic gradient throughout a pipeline is principally obtained by two factors: the discharge pressure at the pumping station and pressure drop due to friction on the pipelines. The discharge pressure at the pumping station is obtained by interpolating on the characteristic curves. With respect to the pressure drop due to friction, there is no standard calculation method in the industry, each pipeline company has its own methods.

The Hazen-Williams equation has been used here. The pressure drop is determined using the following equation

$$
C^{\prime}=3.2310^{6} J^{-0.54} D^{-2.63} f^{0.0081} Q
$$

Where:

C : Coefficient of Hazen- Williams equation

$\mathrm{J}$ : Pressure drop in meters per kilometre

D : Interior diameter of the pipeline in milimetres

f : Product viscosity in centistokes

Q : Flow along a section in cubic metres per hou

This equation gives good results in the Reynolds number range found in oil pipelines.

The advantage of this method against others based on the D'Arcy-Weisbach equation which normally requires iterative solution methods, is its greater speed of calculation. The greatest difficulty when using the Hazen-Williams equation is in determining the constant $\mathrm{C}$ which depends on such factors as the degree of cleansliness and wear and tear on the pipeline.

By looking at the power flow characteristics of the pumps one can see that the pumps power consumption grows slowly with the flow. Therefore from the point of view of energy saving, the best results are obtained when the maximum flow is selected for a particular combination of pumps.

The hydraulic gradient has to exceed the maximum ground elevation transversed by the pipeline. As the hydraulic gradient decreases with flow. there is a maximum flow which is compatible with the hrdraulic gradient exceeding the ground elevation at all points along the pipeline.

In order to calculate the maximum flow in the model, the preassure drop curve for different flows is determined iteratively until the maximum admissable flow is obtained.

As well as the model of the physical characteristics of the pipeline. models of sets of external effects which influence the behaviour mode of the pipeline are necessary. The most importants ones being:

- Variable tank consumption: the chosen model presumes constant tank consumption on working days and zero consumption on the others.

- Description of the contract with the electricity company: At present, the model uses three electricity rates.

- Prefixed time intervals when the pipeline is not in use. 


\section{SIMULATION MODES}

There are two posible ways of working the simulator:

- Automatically. In this case all the actions such as the connection and disconnection of the pumps, the positioning of the valves, etc., are taken by the program in orcler to optimize the electricity bill.

- Manually. The user is in charge of taking all the necessary actions.

As has been mentioned before. only the steady states are considered. To simplify the problem of calculating the evolution of the state of the pipeline with time, a discrete form of simulation has been considered, supposing stationary working as long as there is no qualitative change in the situation. The calculation is considerbly reduced in this way. The main events are:

- The arrival of the interfaces at the components.

- Tank levels reaching limits.

- Changes in electrical tariffs.

- Shut-clown of the system.

- Instalation starting up.

- Periodic events.

The periodic events are introduced to guarantee that the time interval between two consecutive events is small. In this case. the pipeline state does not change substancially from one event to the next. and the pipeline state can be consiclered constant between two consecutives events.

The simulator will function as follows:

a) Read the data on the files and obtain the initial configuration of the system.

b) Adjust the clifferent parameters of the system either manually or using the optimizer. The simulator allows information to become available to the user.

c) Calculate the preassure and flow values throughout the pipeline.

d) Calculate the time in which the next event will take place.

e) With the obtained time calculate the values of the new state of the pipeline.

f) Return to (b).

Another possibility allowed by the simulator is to go to an event previously calculated. In this way, the user can analyse a solution previously obtained. It is also possible to make modifications starting from previous events.

Because of the great amount of data which it is necessary to store of a complete state of the pipeline. only the variables which take a longer computation time and the modifications from one state to another are stored. The rest of the variables are calculated. In this way, a compromise between necessary storage memory and calculating time used has been reached.

Another important feature of these types of tools is the interface with the user. The program uses coloured graphical representations of the state of the pipeline. The graphics are obtained in a completely automatic way using the clata read from the files.

The included graphics are:

- Pipeline topology and representation of the position of the batches.

- Pumping stations.

- Hyclraulic gradient.

- Tanks level at terminals.

- Evolution of the level in the tanks.

The program is also capable of providing print information about clifferent items:

- State of pumping stations.

- State of terminals.

- Pumping station reports.

- Terminal operation reports

- Graphical representation of the hyclraulic gradient.

- Graphical representation of the evolution of the level of the terminals.

\section{OPTIMIZATION ALGORITHM}

The problem consists of finding out how to set the pumps and valves at each time interval in order to deliver the products to the terminal at appropiate rates with minimum electricity costs.

In the following the system will be considered to be operated in the "stripping" mode. That is. each output node is working with an input flow proportional to the amount of product that the terminal has to receive from the current batch. This method of operating is widely considered as the best way of using the transportation capabilities of the pipelines.

Notice that if the stripping mode is used. the amount of product stripped at each terminal clepends on the batch passing at that moment. Therefore, for a given pipeline state the flow at each terminal. and at each bifurcation node. can be computed knowing the flow at the entry node.

The pipeline state can be computed from the volume injected into the entry node and the batch sequence. 
When operating in the stripping mode the future evolution of the system can be computed if the following items are known.

- amount of product which has been pumped (v)

- the time used $(\mathrm{t})$

- the setting of the pumps

- the flow at the entry node.

The state of the system can, therefore, be resumed by the pair $(v, t)$. The control variables are the pump setting and the flow at the entry point. By looking at typical characteristics of pump power consumption - flow, it is clear that the power consumption of the pumps grows slowly with the flow and depends mainly on the pump being connected or not. Therefore, from the power optimization point of view, the best results will be obtained when the maximum flow is chosen.

Taking into account these considerations, the control variables will be a vector of dimension equal to the number of pumps in the system containing a logical value which will indicate if the corresponding pump is active. The number of combinations of the previously mentioned vector can be reduced for pumping stations with identical pumps. In this last case, knowing the number of pumps connected ( in series or parallel ) is enough.

The working space will be the state plane $\mathrm{v}-\mathrm{t}$ and the optimum control problem will consist at determining an optimum trajectory in the plane $\mathrm{v}$ - $\mathrm{t}$ from point $(0,0)$ to point (target-volume,target-time).

The feasible trajectories are constrained by two curves as indicated in figure 1 . The upper one is the curve resulting when pumping the batch sequence using the maximum capabilities of the system. Notice the horizontal pieces of the curve corresponding to intervals where the pumps have to stop either to avoid tank overflow or due to maintenance shut-down of the system. The lower curve is computed taking into account the minimum pumped amount at each interval in order to satisfy the needs and taking into account the pumping capabilities of the system.

The optimization algorithm uses the cost function:

$$
f\left(x_{v, t}\right)=g\left(x_{v, t}\right)+h\left(x_{v, t}\right)
$$

where $x_{v, t}$ is the state vector and consists of a pair (volume,time), $g\left(x_{v, t}\right)$ is a function representing the minimum cost from the origin to node $x_{v, t}$ and $h\left(x_{v, t}\right)$ is a function that estimates the minimum cost from $x_{v, t}$ to a goal node. That is, $f\left(x_{v, t}\right)$ is an estimate of the cost of the minimal cost path constrained to go through node $x_{v, t}$.

The optimization algorithm works in two steps. In the first one the function $h\left(x_{v, t}\right)$ is computed on a grid by using Bellman's Optimality Principle and in the second one the optimum path is found by using this function and an accurate simulation of the transportation system.

In the first step of the algorithm, function $h\left(x_{v, t}\right)$ is made equal to zero for all $x_{v, t}$ with volume equal or greater than the target volume. The next step for estimating the function $h\left(x_{v, t}\right)$ is to compute all states $x_{v_{1}, t_{1}}$ from where $x_{v, t}$ can be reached. Notice that if the volume difference $\Delta v=v-v_{1}$ is small, the pipe state will be practically the same. Therefore, for a given pump combination the flow will be very similar and can be considered constant.

In order to avoid the combinatorial explosion, a procedure

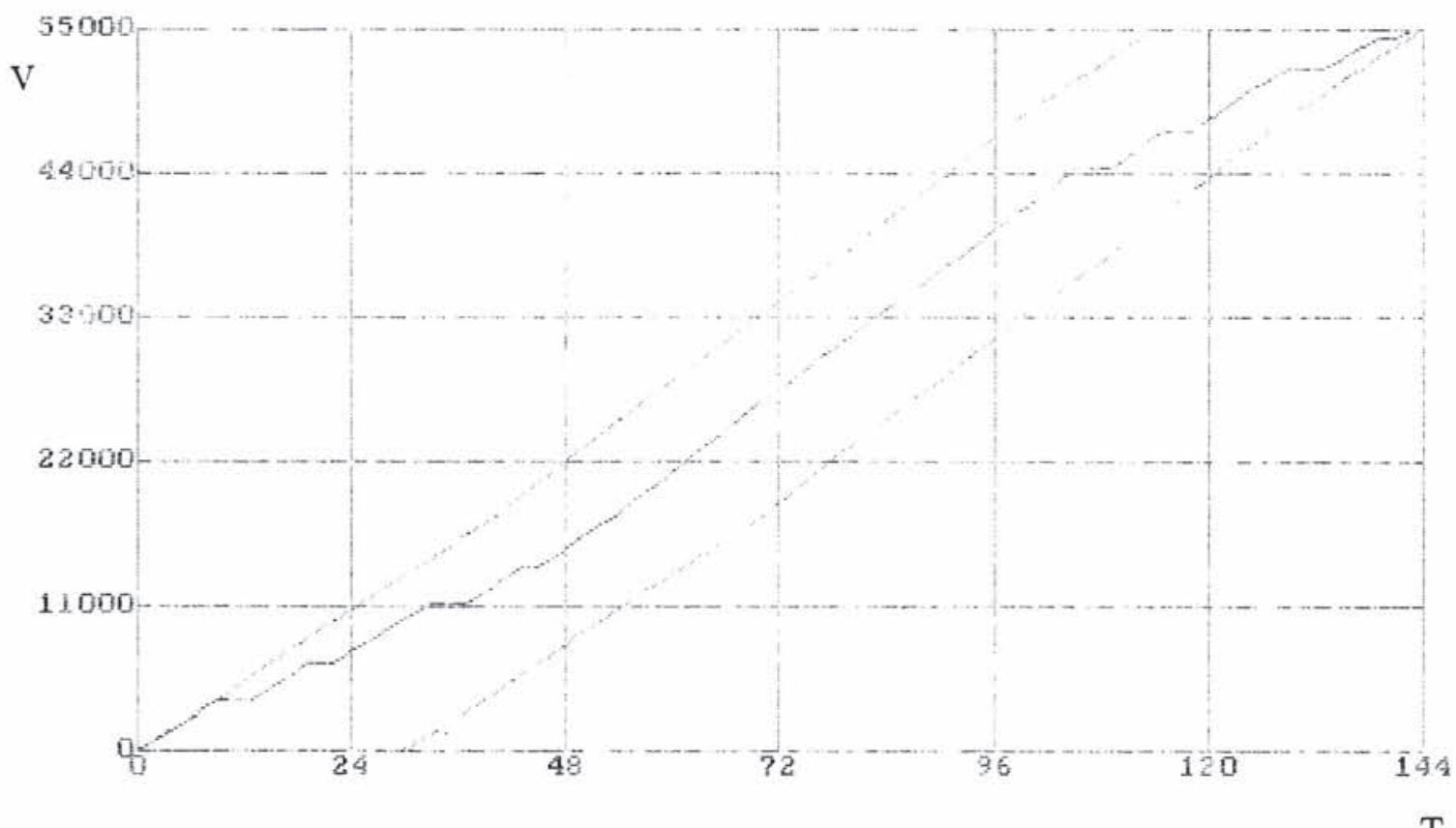

Fig. 1. Feasible Optimization Space State and Optimum trajectory. 


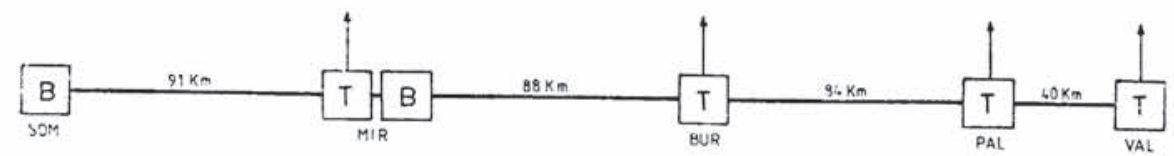

Fig. 2. BILVA Pipeline.

for eliminating states is used. At first, the procedure eliminates the trivial states, and then a set of heuristic rules are used to decide which states are "the best" for the next step of the algorithm.

A state $x_{v, t}$ is considered to be trivial if there is another state $x_{v, t}$, with

$t_{i} \geq t$ and

$h\left(x_{v, t}\right) \geq h\left(x_{v, t_{1}}\right)$.

Notice that state $x_{v, t}$ could be reached from $x_{v, t_{1}}$ by switching off all the pumps between $t_{i}$ and $t$ (with cost zero) and the function $h\left(x_{v, t}\right)$ will therefore be equal to $h\left(x_{v, t_{1}}\right)$.

Once all trivial state have been eliminated, a second elimination procedure takes place in orcler to obtain a feasible amount of states for the next step.

Once the function $h\left(x_{v, t}\right)$ has been computed over the grid, the second step of the algorithm can be carried out. It consists of computing, at each interval and for all possible pumping combinations, the accumulated cost to the next event $g\left(x_{v, t}\right)$ plus the estimated cost to the goal node $h\left(x_{v, t}\right)$. The function $h\left(x_{v, t}\right)$ is calculated by a linear interpolation in the grid computed in the first part of the algorithm.

The accumulated cost is computed by using an accurate simulation of the pipeline network. It involves the computation of pressure drops. flow, power consumption etc. and it takes into account the non-linear characteristics of pumps and pipelines as well as different electricity tariffs throughout the day:

\section{APPLICATION TO OIL PIPELINES}

The simulator and the optimization algorithm has been included in a program developed for CAMPSA. The program can be applied to any pipeline transportation system with only one entry node. The algorithm has been tested with the model of an oil pipeline shown in figure 2, corresponding to the pipeline BILVA, running in Northern Spain.

The pipeline has four terminals and two pump stations. The first one, shown in figure 3 , has three booster pumps operating in parallel and four main pumps operating in series. The second one has three main pumps operating in series. The three booster pumps have identical characteristics, and so do the main pumps at the first pumping station. The three main pumps at the second pumping station are also identical.

Five types of products, two types of gasoline, two types of cliesel oil and kerosene, were considered.

The algorithm has been tested with different cases. Table I shows one of the batch sequences used in the tests. Figure 1 corresponds to the solution in a case covering the batch schedule during ten days. Notice that the upper curve has a few horizontal pieces corresponding to points where the tanks would overflow if the pumping continued at maximum flow.

The different slopes in the curves correspond to different flows due to different pumping conditions as fluids in the

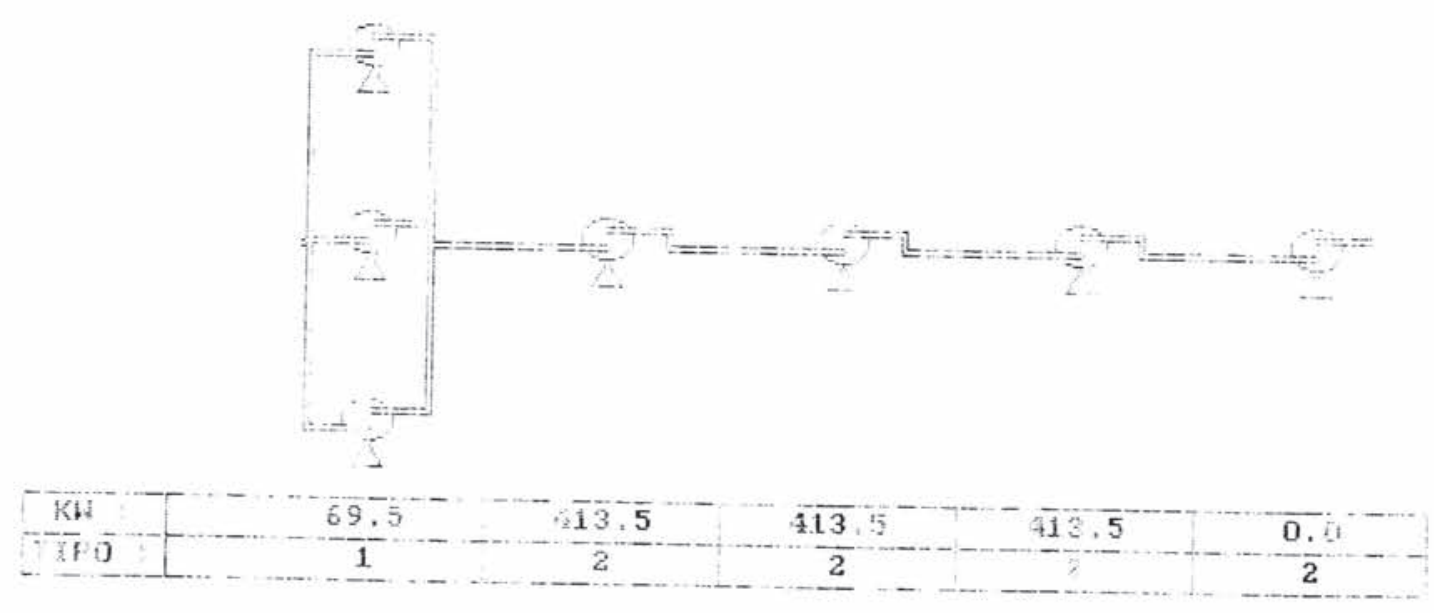

Fig. 3. Pumping Station. 
TABLE 1. Batch Sequence.

\begin{tabular}{|c|c|c|c|c|c|}
\hline NAME & UOLUAE $\left(H^{2}\right)$ & MIRAND $(\%)$ & BURGOS $(\%)$ & PALENC ( $\%)$ & UALLAD (\%) \\
\hline $50 / 025 / 97$ & 10200 & 15.68 & 7.84 & 0.00 & 76.48 \\
\hline so/011/92 & 7800 & 43.59 & 5.13 & 0.00 & 51.28 \\
\hline $30 / 026 / 97$ & 25000 & 29.60 & 10.00 & 0.00 & 54.40 \\
\hline SO/029/RD & 200 & 0.00 & 0.20 & 0.00 & 0.00 \\
\hline$S 0 / 026 / G A$ & 11300 & 8.80 & 1.50 & 6.40 & 91.20 \\
\hline
\end{tabular}

TABLE 2. Optimal Solutions.

\begin{tabular}{|c|c|c|c|}
\hline INITIAL DAIE & TARGET TIME (HOURS) & TARIEET JULUHE $\left\langle M^{3}\right\rangle$ & CSST (PTS.) \\
\hline $0: 00 \quad 1 / 6 / 89$ & 120 & 55000 & $1.243 .22 ?$ \\
\hline $0: 00 \quad 1 / 6 / 39$ & 144 & 55000 & 1.095 .260 \\
\hline $0: 00 \quad 1 / 6 / 89$ & 168 & 55000 & 1.019 .276 \\
\hline $0: 00 \quad 1 / 6 / 89$ & 192 & 55000 & 981.075 \\
\hline $0: 00 \quad 1 / 6 / 89$ & 240 & 55000 & $943.390^{\circ}$ \\
\hline $0: 80 \quad 1 / 6 / 89$ & 288 & 55000 & 834.113 \\
\hline $0: 00 \quad 1 / 6 / 89$ & 360 & 55000 & 832.980 \\
\hline
\end{tabular}

pipeline and flows in the output terminals change with state.

The lower curve corresponds, as mentioned before, to the minimum volume that has to be pumped at each time interval in order to satisfy the consumers' needs. Notice that the pipeline is kept switch off and that the maximum flow is used in order to pump the target volume in the prescribed time.

The thicker line corresponds to the optimum trajectory. It can be seen that the solution involves shutting down all pumps during peak hours, and using high flow combinations during valley periods.

Good economical results have been achieved using the algorithm in the management of the oil pipeline. Table II shows the clifferent solutions obtained when using different target times. As the target time increases the products can be transported during off-peak hours and the cost therefore decreases.

The energy cost has been reduced in approximately 20 per cent from an initial solution.

\section{CONCLUSIONS}

A simulator of oil pipeline networks has been presented. The simulator has been cleveloped to help schedulers to operate the pipeline and inclucles an algorithm for the optimization of the power costs. The algorithm uses a function that measures the estimated minimum cost to the goal node and an accurate simulation of the transportation system. The algorithm has been tested with success in an oil pipeline in Northen Spain.

\section{ACKNOWLEDGEMENT}

The authors would like to acknowledge the finantial support of CAMPSA for this work and also to thank S. Heras and R. LLorente of the Departamento de Oleoductos y Operaciones of CAMPSA for their valuable comments. Further thanks is clue to F. J. Vidal. C. del Valle, J. Gómez and R. Millán for their collaboration.

\section{REFERENCES}

Basavaraj. B.H. (1984) Cut pipeline power cost by demand control. Oil \& Gas Journal. Jan, pp. 88-90.

Burham C.G. (1983) Rate Schedule Application - Off Peak Operation of Pump Station. Proc. of the Pipeline Engineering Symposium. p1. 11-16, Houston. Texas.

Coulbeck B. and M. Sterling. (1978) Optimizated Control of Water Distribution Systems. Proc. IEEE. Vol. 122, N. 2 .

Kilempous R. J. Kotwosky. J. Nikodem and J. Clasiewicz. (1986). Water Distribution Systems. Components, Instru. ments and Techniques for Low Cost Automation \& Applications. pp. 541-551. Valencia (Spain).

Miyaoka S.. M. Funabashi. (19\$4) Optimal Control of Water Distribution Systems by Network Flow Theory. IEEE Transaction on Automatic Control. Vol. AC-29, N. 4. pp. 303-311.

Camacho E.F.. M.A. Ridao and J.A. Ternero (1989) Power Optimization of Multi-fluid Transportation Systems. Proc. of INCOM 89. Madricl (Spain). 